\title{
Homophilic complex formation of CDCP1 via the extracellular CUB2 domain facilitates SFK activation and promotes cancer cell migration
}

\author{
TADASHI SAWAYAMA ${ }^{1}$, KATSUHIKO NAKASHIMA ${ }^{2}$, TOHRU ICHIMURA ${ }^{1}$, \\ RYUICHI SAKAI ${ }^{2,3}$ and TAKAMASA UEKITA ${ }^{1}$ \\ ${ }^{1}$ Department of Applied Chemistry, National Defense Academy, Yokosuka 23908686; \\ ${ }^{2}$ Division of Refractory and Advanced Cancer, National Cancer Center Research Institute, Tokyo 105-0045; \\ ${ }^{3}$ Division of Biochemistry, Kitasato University School of Medicine, Kanagawa 252-0374, Japan
}

Received December 10, 2018; Accepted June 28, 2019

DOI: $10.3892 /$ or.2019.7271

\begin{abstract}
CUB domain-containing protein 1 (CDCP1) is phosphorylated by Src family kinases (SFK), and is thought to serve an important role in tumor metastasis through downstream signaling subsequent to its interaction with protein kinase $\mathrm{C} \delta$. The present study investigated the mechanisms of activation for CDCP1 signaling, and demonstrated that CDCP1 is able to activate SFK via a homophilic complex of the extracellular complement $\mathrm{C} 1 \mathrm{r} / \mathrm{C} 1 \mathrm{~s}$, urchin embryonic growth factor, bone morphogenetic protein 1 (CUB) 2 domain. Deletion of the extracellular CDCP1 region abolished homophilic complex formation of CDCP1 and the ability to promote cancer cell migration. When the culture medium was supplemented with recombinant CUB2 domain protein fused with maltose binding protein (rMBP-CUB2), CDCP1 homophilic complex formation was effectively inhibited. rMBP-CUB2 also inhibited SFK activation and the migratory capacity of invasive human lung adenocarcinoma A549 cells, and human pancreatic BxPC3 cells. These findings demonstrated a novel function for the extracellular CUB2 domain of
\end{abstract}

Correspondence to: Dr Takamasa Uekita, Department of Applied Chemistry, National Defense Academy, 1-10-20 Hashirimizu, Yokosuka, Kanagawa 23908686, Japan

E-mail: tuekita@nda.ac.jp

Abbreviations: CDCP1, CUB domain-containing protein 1; CDCP1res-F, FLAG-tagged CDCP1 rescue; CDCP1res-HA, HA-tagged CDCP1 rescue; CUB, complement $\mathrm{Clr} / \mathrm{C} 1 \mathrm{~s}$, urchin embryonic growth factor, bone morphogenetic protein 1; ECD, extracellular domain; $\triangle E C D-F$, extracellular domain deletion mutant of CDCP1 with a FLAG tag at the C-terminus; rMBP-CUB2/3, recombinant CUB2 or CUB3 domain proteins combined with maltose binding protein; SFK, Src family kinase; SH2, Src homology 2

Key words: CUB domain-containing protein 1, cancer, homophilic complex, Src activation, cell migration
CDCP1, promoting cancer cell migration via SFK activation on the plasma membrane. It was also indicated that the region blocking the homophilic binding site may be a potential therapeutic target against CDCP1-dependent tumor invasion.

\section{Introduction}

Src family kinases (SFKs) comprise the largest family of non-receptor tyrosine kinases (1). Activated SFKs initiate numerous signaling pathways; under the control of extracellular stimuli, these pathways serve important roles in various cellular functions, including proliferation, migration and survival (2). SFK activation results in the phosphorylation of $\mathrm{Tyr}^{416}$ in the kinase domain (3), and SFK activity is regulated by a phosphotyrosine ligand with a higher affinity for the $\mathrm{SH} 2$ domain (1). Studies have demonstrated increased SFK activity in a number of human cancers (4), thus the analysis of SFK activation is important to understand tumor progression.

CUB domain-containing protein 1 (CDCP1) is a type I transmembrane protein, also known as SIMA135 (5), gp140 (6) and Trask (7). Initially, CDCP1 was reported as being predominantly expressed in colorectal cancers (8). However, in an investigation of the phosphoprotein associated with anchorage independence of lung adenocarcinoma cells, it was observed that CDCP1 saved a principal role as the SH2 domain-binding phosphoprotein of SFK in anoikis inhibition of metastatic tumor cells (9). SFK activation results in the phosphorylation of tyrosine residues in CDCP1; when this occurs in the intracellular domain, protein kinase $\mathrm{C} \delta(\mathrm{PKC} \delta)$ is recruited to the plasma membrane, where it subsequently undergoes specific phosphorylation by SFK at $\mathrm{Tyr}^{311}(9-11)$. This SFK-CDCP1-PKCס signaling is involved in the regulation of anoikis resistance (9), cell migration (12), extracellular matrix degradation (13) and invasion (14).

CDCP1 possesses three complement $\mathrm{C} 1 \mathrm{r} / \mathrm{C} 1 \mathrm{~s}$, urchin embryonic growth factor bone morphogenetic protein 1 (CUB) domains in its extracellular domain (ECD), which may be involved in protein-protein interactions (15-17). Therefore, CDCP1 may form a homophilic complex at the plasma membrane through its ECD. CUB1, the domain 
located furthest from the transmembrane domain, is cleaved and released, and has been detected in the urine of patients with cancer $(18,19)$. CDCP1 cleavage in the ECD induces cell migration in triple-negative breast cancer cells (18). These findings demonstrated that the extracellular CUB domains of CDCP1 potentially regulate tumor cell migration by promoting CDCP1 signaling; however, the functional mechanism of this activation is not yet understood. The present study investigated how CUB domains stimulate CDCP1 signaling. Utilizing recombinant proteins, it was demonstrated for the first time that the extracellular CUB2 domain of CDCP1 is the formation site of the CDCP1 homophilic complex. This provides novel information on the role of the CUB2 domain in regulating intracellular CDCP1 signaling.

\section{Materials and methods}

Antibodies. Anti-HA (Y-11; cat. no. sc-805; 1:300), anti-HA-horseradish peroxidase(HRP)-conjugated (Y-11 HRP; cat. no. sc-805 HRP; 1:500), anti-c-Src (SRC2; cat. no. sc-18; 1:4,000) and anti-PKCס (C-20; cat. no. sc-937; 1:4,000) antibodies were purchased from Santa Cruz Biotechnology. Inc. Anti-FLAG M2 fluorescein isothiocyanate-conjugated (cat. no. F4049; 1:600), anti-FLAG M2 peroxidase-conjugated specific (cat. no. A8592; 1:4,000) and anti- $\alpha$ tubulin (cat. no. T5168; 1:10,000) antibodies were purchased from Sigma-Aldrich (Merck KGaA). An anti-maltose-binding protein (MBP) antibody (cat. no. E8032 1:10,000) was purchased from New England BioLabs, Inc. Anti-phospho-Src family $\left(\mathrm{Tyr}^{416}\right.$, and $\left.2101 ; 1: 2,000\right)$ and anti-phospho-PKC $\delta$ ( $\mathrm{Tyr}^{311}$; cat. no. $\left.2055 ; 1: 2,000\right)$ antibodies were purchased from Cell Signaling Technology, Inc. HRP-conjugated anti-mouse IgG (cat. no. NA931V; 1:4,000) and anti-rabbit IgG (cat.no. NA934V; 1:4,000) antibodies were purchased from GE Healthcare. Alexa Fluor 488 goat anti-mouse (cat. no. A11011; 1:800) and Alexa Fluor 546 goat anti-rabbit (cat. no. A11010; 1:800) antibodies were purchased from Thermo Fisher Scientific, Inc. Rabbit polyclonal anti-CDCP1 antibody was prepared as described previously (9).

Expression plasmids. pcDNA3.1 expression plasmids (Thermo Fisher Scientific, Inc.,) encoding human CDCP1 with a C-terminal FLAG or HA tag, and the CDCP1 rescue sequence that introduces silent mutations that are not suppressed by CDCP1 siRNA (CDCP1res-F and CDCP1res-HA, respectively), have been described previously (13). The ECD-deleted mutant plasmid with C-terminal FLAG tag ( $\triangle \mathrm{ECD}-\mathrm{F})$ was generated by inverse PCR using the KOD-Plus-Mutagenesis kit (Toyobo Life science) per the manufacturer's protocol.

A system for the stable, siRNA-induced suppression of CDCP1 expression was constructed using the Block IT Pol II miRNA Expression Vector kit (Invitrogen; Thermo Fisher Scientific, Inc.,) according to the manufacturer's instructions. The following primers were used to generate the CDCP1 miR RNAi plasmid (miCDCP1): Forward, 5'-TGCTGAATGTTGCTTTCTCGTGGCAGGTTTTGGC CACTGACTGACCTGCCACGAAAGCAAGATT-3', and reverse 5'-CCTGAATGTTGCTTTCGTGGCAGGTCA GTCAGTGGCCAAAACCTGCCACGAGAAAGCAAC ATTC-3'. The LacZ miR RNAi plasmid was selected as the negative control, and was constructed using the following primers: Forward, 5'-TGCTGAAATCGCTGATTTGTG TAGTCGTTTTGGCCACTGACTGACGACTACACATCA GCGATTT-3', and reverse, 5'-CCTGAAATCGCTGAT GTGTAGTCGTCAGTCAGTGGCCAAAACGACTAC ACAAATCAGCGATTTC-3'.

Cell culture and transfection. Human lung adenocarcinoma A549 and human pancreatic cancer BxPC3 cell lines were cultured in RPMI 1640 (Sigma-Aldrich; Merck KGaA) supplemented with $10 \%$ fetal bovine serum (FBS; Gibco; Thermo Fisher Scientific, Inc.) at $37^{\circ} \mathrm{C}, 5 \% \mathrm{CO}_{2}$. A549 miCDCP1 cells with suppressed CDCP1 expression, and A549 miLacZ cells (the control cells from the Block IT Pol II miRNA Expression Vector kit) were used as described previously (9).

For transfection, cells were seeded at $5 \times 10^{6}$ cells $/ 10-\mathrm{cm}$ dish or $1 \times 10^{5}$ cells/well of a 24 -well plate, prior to a $24-\mathrm{h}$ incubation period. The cells were transfected with the expression plasmids (CDCP1res-F, $5 \mathrm{mg}$; CDCP1res-HA, $5 \mu \mathrm{g}$; and $\Delta \mathrm{ECD}-\mathrm{F}, 1 \mu \mathrm{g}$ ) using Lipofectamine ${ }^{\mathrm{TM}} 2000$ (Invitrogen; Thermo Fisher Scientific, Inc.) according to the manufacturer's instructions. The transfected cells were selected in the presence of $800 \mu \mathrm{g} / \mathrm{ml} \mathrm{G} 418$ sulfate (EMD/Merck KGaA) for one month prior to experimentation.

Immunofluorescence staining. For immunostaining, cells cultured on cover glasses were washed with phosphate-buffered saline (PBS) at $37^{\circ} \mathrm{C}$ and fixed with $4 \%$ paraformaldehyde in PBS for 10 min at room temperature. The cells were washed again with PBS and permeabilized with $0.25 \%$ Triton X-100 in PBS. The cells were the blocked with Blocking One (Nacalai Tesque, Inc.,) for $40 \mathrm{~min}$, and co-localization was analyzed using a confocal laser-scanning microscope (FLUOVIEW FV10i) and the FV10-ASW software ver. 4.1 'Co-Localization' (Olympus Corporation). At a certain threshold, the FV10-ASW software converts the pixels of the area co-stained with two fluorescent substances into a white dot; the ratio of white dot pixels to total pixels is then determined, and the pixel rate corresponds to the co-localization area value.

Western blotting and immunoprecipitation. Cell lysates were prepared in PLC buffer [10 mM Tris- $\mathrm{HCl}(\mathrm{pH} 7.5), 5 \mathrm{mM}$ EGTA, $150 \mathrm{mM} \mathrm{NaCl}, 1 \%$ Triton $\mathrm{X}-100,10 \%$ glycerol and $1 \mathrm{mM}$ sodium orthovanadate]. The lysates were centrifuged at $20,630 \mathrm{x} \mathrm{g}$ for $20 \mathrm{~min}$ at $4^{\circ} \mathrm{C}$, and the supernatants were collected. Protein concentrations were measured using a BCA Protein Assay kit (Pierce; Thermo Fisher Scientific, Inc.).

For immunoprecipitation, the lysates $(1 \mu \mathrm{g} / \mu \mathrm{l} ; 350 \mu \mathrm{l})$ were incubated with anti-FLAG M2 affinity gel $(20 \mu \mathrm{l})$ or anti-HA antibody conjugated with Protein G Sepharose 4 Fast Flow gel $(30 \mu \mathrm{l})$ on ice for $1 \mathrm{~h}$. The anti-FLAG M2-protein or anti-HA-protein complexes were then harvested and washed three times with PLC lysis buffer. The total amount of each immunoprecipitated sample was separated by $8 \%$ SDS-PAGE gel and visualized using western blotting.

For western blotting, the samples were transferred to polyvinylidene difluoride (PVDF) membranes (Immobilon-P; EMD Millipore). After blocking with blocking buffer (Blocking One; Nacalai Tesque) the membranes were probed with primary antibodies (anti-CDCP1, anti- $\alpha$ tubulin, anti-FLAG M2 
peroxidase-conjugated, anti-HA, anti-HA-HRP-conjugated, anti-MBP, anti-c-Src, Anti-phospho-Src family, anti-PKCס and anti-phospho-PKCס) at room temperature for $1 \mathrm{~h}$ to detect the indicated proteins. After washing three times with TBS-T (0.05\% Tween-20 in TBS), the membranes were further probed with the corresponding secondary antibodies at room temperature for $30 \mathrm{~min}$. The blots were visualized using the Western Lightning Plus-ECL kit (PerkinElmer, Inc.) and images were captured with the myECL imager (Thermo Fisher Scientific, Inc.). The proteins were subsequently quantified using ImageJ software, version 1.50i (National Institutes of Health).

Scratch wound-healing assay. Toinvestigate the wound-healing effect, A549 miCDCP1 cells transfected with CDCP1res-F, $\triangle \mathrm{ECD}-\mathrm{F}$ or mock (pcDNA3.1) plasmids were seeded in RPMI $(10 \%$ FBS $)$ at $1 \times 10^{6}$ cells/well in 6 -well, and incubated until confluent. Each monolayer was scratched to create a wound of $\sim 600 \mu \mathrm{m}$. After washing three times with PBS to remove cell debris, $5 \mathrm{ml}$ RPMI (10\% FBS) was added to each well. Images were captured by phase-contrast microscopy (BZ-X 710; Keyence Corporation) at 0 and $12 \mathrm{~h}$ for BxPC3 cells, and after $24 \mathrm{~h}$ for A549 cells. Wound recovery was measured with the BZ-X analyzer. Cell migration was evaluated as the mean of the migration length in three independent experiments. BxPC3 cell migration was analyzed in the same manner, with the only difference being the timing of image capture (at 0 and $12 \mathrm{~h}$ ).

$M B P$ protein expression and far-western blotting. To create MBP constructs, the amplified CUB2 and CUB3 domain sequences of CDCP1 were inserted into the $p M A L-c 5 x$ expression vector (New England BioLabs, Inc.). PCR was performed using the following primers: rMBP-CUB2 forward, 5'-CGC ATATGTGCACAGACCACCGGTACTGC-3', and reverse, 5'-GCGAATTCAACGCCTTCCTCTTTGAAATAAG-3'; and rMBP-CUB3 forward, 5'-GCCATATGGAGGAAGGCGTTT TCACGGTGAC-3', and reverse, 5'-CGGAATTCTGGGGTA AGTGTCACCGAGAAGAG-3'. Protein expression and affinity purification with amylose resin were performed in accordance with the manufacturer's protocol.

For far-western blotting, each rMBP protein $(3 \mu \mathrm{g})$ was blotted onto a PVDF membrane. After blocking with blocking buffer for $1 \mathrm{~h}$, each cell lysate expressing CDCP1res-F or $\Delta$ ECD-F $(10 \mu \mathrm{g} / \mathrm{ml}$ with TBS-Tween 20$)$ was incubated with the membrane for $1 \mathrm{~h}$. After washing three times with TBS-Tween 20, the membrane was used for western blotting.

Statistical analysis. All data are presented as the mean \pm standard deviation; ANOVA followed by Tukey's test was used for multiple comparisons among sample groups. $\mathrm{P}<0.05$ was considered to indicate a statistically significant difference.

\section{Results}

CDCP1 forms a homophilic complex via the ECD. A previous study indicated that CDCP1 was capable of forming dimers within the cell (20). To verify this phenomenon, FLAG-tagged CDCP1 (CDCP1res-F) and HA-tagged CDCP1 (CDCP1res-HA) (Fig. 1A) were co-expressed in A549 miCDCP1 cells, where endogenous CDCP1 expression was stably suppressed (26.1 $44.0 \%$; Fig. 1B). CDCP1res-F was immunoprecipitated with a anti-FLAG M2 antibody. As shown in Fig. 1C, CDCP1res-HA was co-immunoprecipitated with CDCP1res-F (lane 5), and the same result was observed by immunoprecipitation with the anti-HA antibody (lane 8). This indicated that differentially tagged CDCP1 molecules formed a complex with each other. To determine whether the ECD of CDCP1 was important for complex formation, $\triangle \mathrm{ECD}-\mathrm{F}$ (which still contained the $\mathrm{N}$-terminal signal sequence and is expressed at the cell membrane) was co-expressed with CDCP1res-HA (Fig. 1A) and subjected to immunoprecipitation and western blotting with anti-FLAG M2 and anti-HA antibodies. CDCP1res-HA was associated with CDCP1res-F; however, by comparison, less association was detected with CDCP1res-HA and $\triangle \mathrm{ECD}-\mathrm{F}$ (Fig. 1C, lanes 6 and 9). In addition, two control IgG antibodies did not immunoprecipitate with CDCP1res-F and CDCP1res-HA (Fig. 1C, lanes 11 and 14).

CDCP1 homophilic complex formation on cells was also investigated. CDCP1res-HA and either CDCP1res-F or $\triangle E C D-F$ was co-expressed in A549 miCDCP1 cells, and localization was examined by immunostaining. CDCP1res-F and CDCP1res-HA were detected on the plasma membrane and ruffling edge (Fig. 2A, inserts a, b, and f). CDCP1res-F and CDCP1res-HA were also primarily co-localized at the plasma membrane and the periphery of cells (Fig. 2A, insert d). Although $\triangle E C D-F$ was expressed around the plasma membrane (Fig. 2A, insert e), it was localized mainly at a region of the cell surface that was different from the region at which CDCP1res-HA was localized (Fig. 2A, insert h). To monitor the level of co-localization of CDCP1 variants at the cell surface, the co-localization areas were quantified. The co-localization of each CDCP1 variant on the cell membrane is shown as the area of white dots detected by FV10-ASW software (Fig. 2B, inserts a and b). The co-localization area of CDCP1res-F and CDCP1res-HA $(6.89+1.95 \%)$ was greater than that of $\triangle E C D-F$ and CDCP1res-HA $(1.63+0.76 \%)$ (Fig. 2B). Taken together, these results suggested that the ECD is required for CDCP1 homophilic complex formation at the cell surface.

CDCP1 ECD regulates lung cancer cell migration. Previous reports have suggested that CDCP1 is important for the regulation of cancer cell migration (13). It was thus hypothesized that CDCP1 formed a homophilic complex via the ECD on the cell surface to regulate cell migration. A scratch wound-healing assay was used to assess the significance of the CDCP1 ECD in cancer cell migration (Fig. 3A). Cells with CDCP1 suppression exhibited significantly reduced migratory properties, compared with CDCP1-expressing cells (Fig. 3B, miLacZ vs. miCDCP1 Mock vector), as supported by a previous report (13). For the rescue experiments, CDCP1res-F and $\triangle E C D-F$ vectors were transfected into A549 miCDCP1 cells. Cell migration was recovered by the $\mathrm{CDCP} 1$ res-F vector, but not by the $\triangle \mathrm{ECD}-\mathrm{F}$ mutant vector (Fig. 3B). This result demonstrated that the CDCP1 ECD is required for cancer cell migration.

CDCP1 forms a homophilic complex via the CUB2 domain. Given the reported importance of CUB1 domain release in CDCP1 dimerization $(18,21)$, it was hypothesized that the extra- 
A

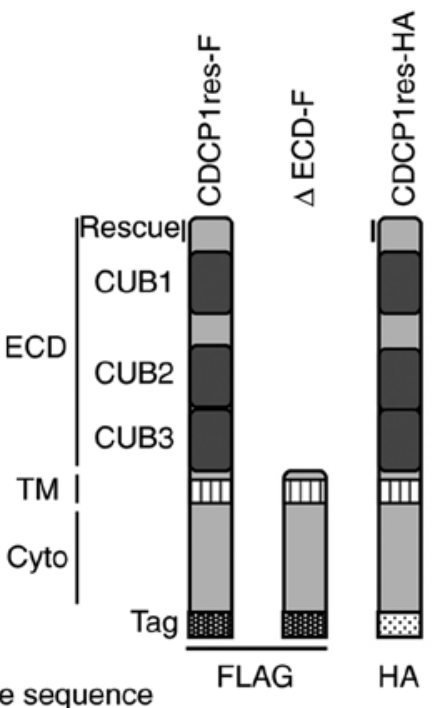

\begin{tabular}{l} 
Rescue sequence \\
\hline
\end{tabular}

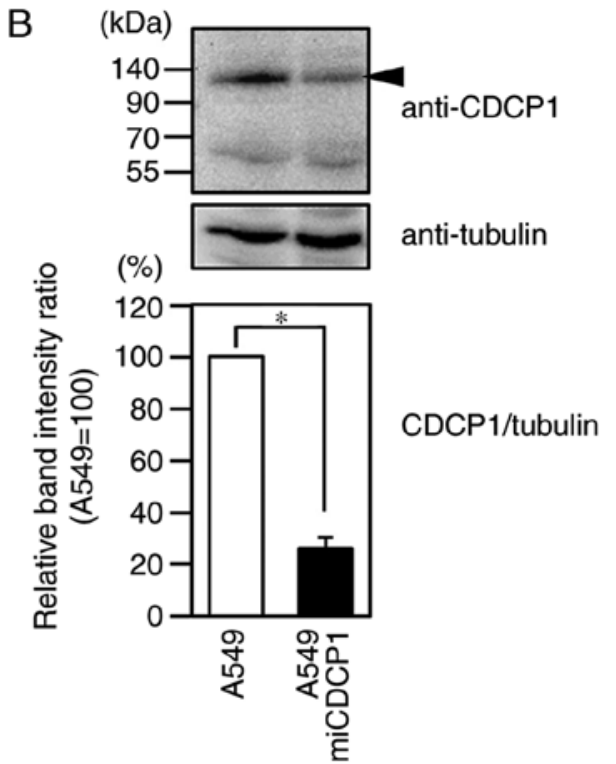

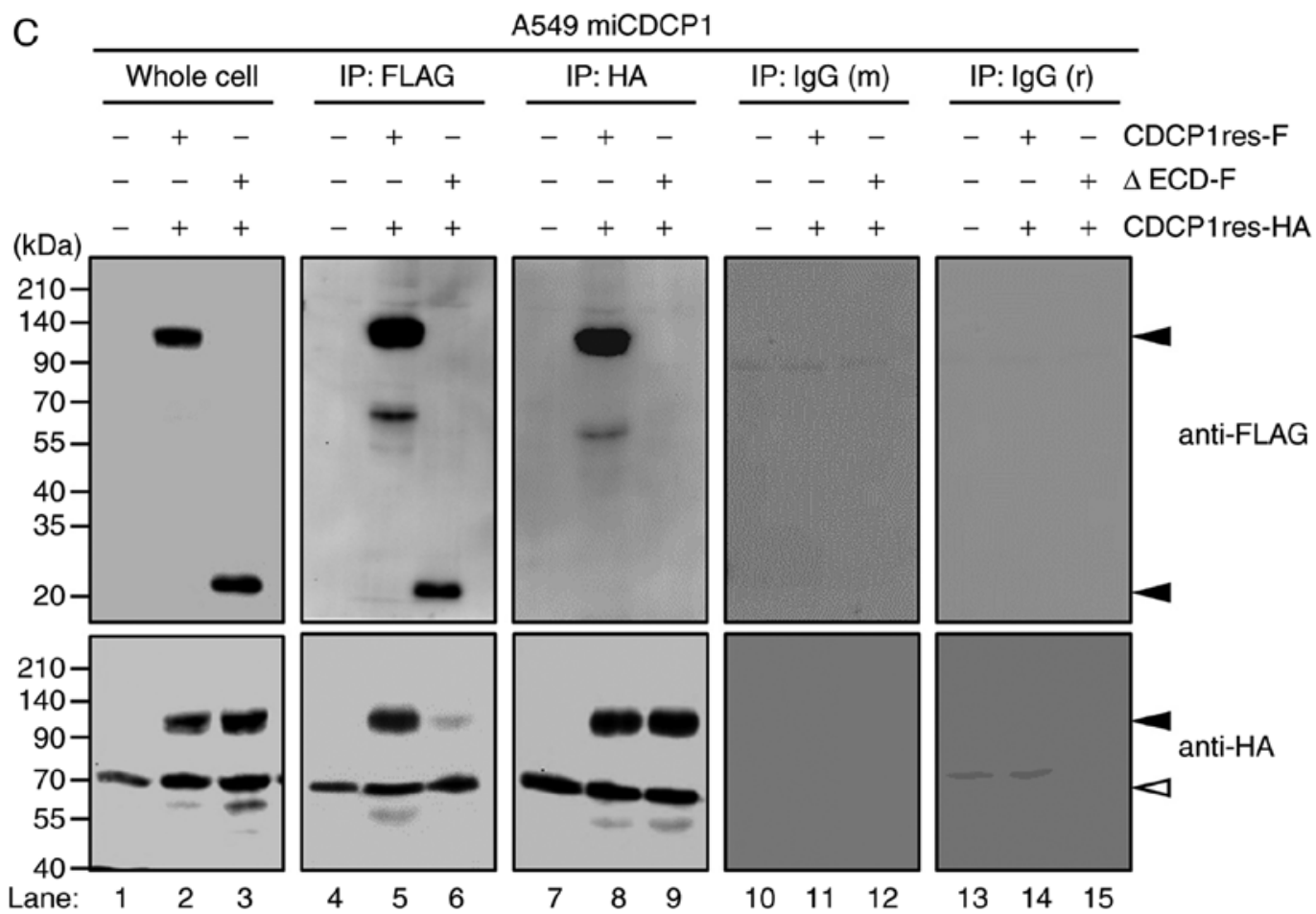

Figure 1. CDCP1 forms a homophilic complex via its ECD. (A) Schematic of the CDCP1 variants. CDCP1res-F and CDCP1res-HA (135 kDa) include the CUB domain in the ECD. $\triangle \mathrm{ECD}-\mathrm{F}(20 \mathrm{kDa})$ lacks the ECD, but contains the signal sequence. The FLAG or HA tag is attached to the C-terminus. Rescue, target CDCP1 DNA sequence and the rescue mutant sequence that is not suppressed by CDCP1 small interfering RNA (13). (B) Stable suppression of CDCP1 in A549 miCDCP1 cells. Cells were cultured in RPMI 1640 with $800 \mu \mathrm{g} / \mathrm{ml} \mathrm{G} 418$ sulfate. Cell lysates were subjected to western blotting using an anti-CDCP1 antibody. Tubulin served as an internal control. The suppression ratio is shown in the bar graph. Mean \pm standard deviation; $n=3$ for each bar; " $P<0.05$, tukey's test. (C) A549 miCDCP1 cell lysates co-expressing either CDCP1res-F and CDCP1res-HA or $\triangle E C D-F$ and CDCP1res-HA were immunoprecipitated with anti-FLAG M2, anti-HA, control IgG (m) or IgG (r) antibodies. Whole cell lysates and precipitates were subjected to immunoblotting with anti-FLAG and anti-HA antibodies. Black arrowheads indicate CDCP1 variants. White arrowheads indicate nonspecific bands. CDCP1, CUB domain-containing protein; ECD, extracellular domain; CUB, complement $\mathrm{Clr} / \mathrm{C} 1 \mathrm{~s}$, urchin embryonic growth factor, bone morphogenetic protein 1; TM, transmembrane domain; Cyto, cytoplasmic domain; bp, base pair; a.a., amino acids; IgG (m), control mouse IgG; IgG (r), control rabbit IgG; -F, FLAG-tagged; -HA, HA-tagged.

cellular CUB2 and/or CUB3 domains of CDCP1 served a role in the regulation of homophilic complex formation. To test this hypothesis, recombinant CUB2 or CUB3 domains were fused with the MBP (rMBP; rMBP-CUB2 and rMBP-CUB3, respec- tively) (Fig. 4A). The rMBP fusion proteins (3 $\mu \mathrm{g}$ each) were immobilized onto a PVDF membrane (Fig. 4B, rMBP proteins) and blotted with A549 miCDCP1 cell lysates expressing CDCP1res-F or $\triangle E C D-F$. Each protein was visualized 

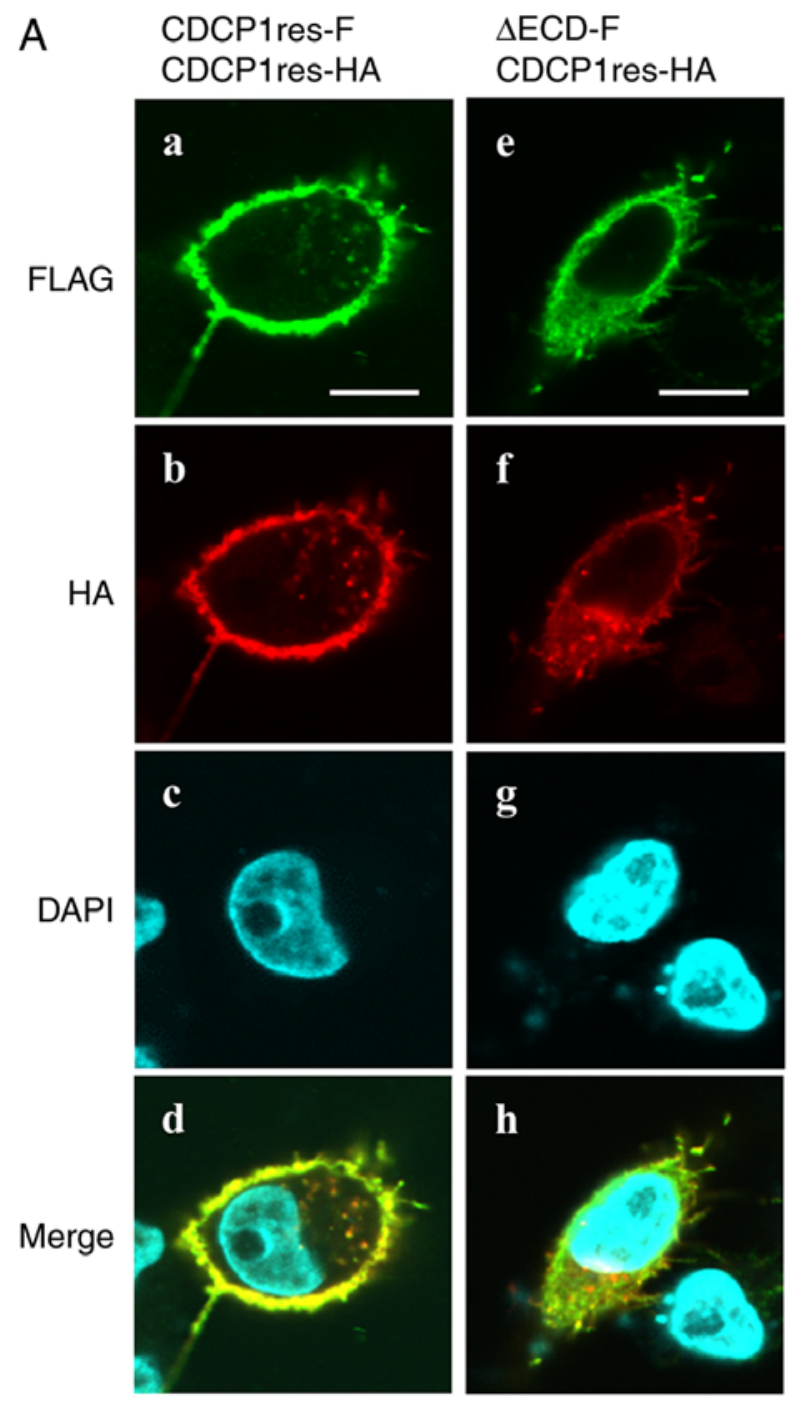

B

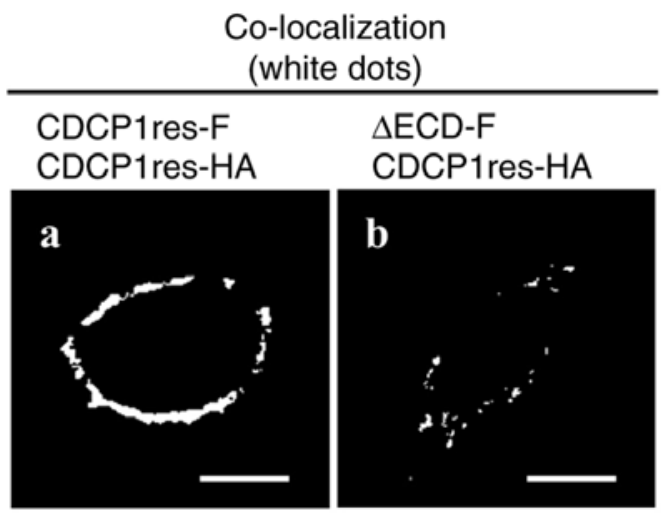

(\%)

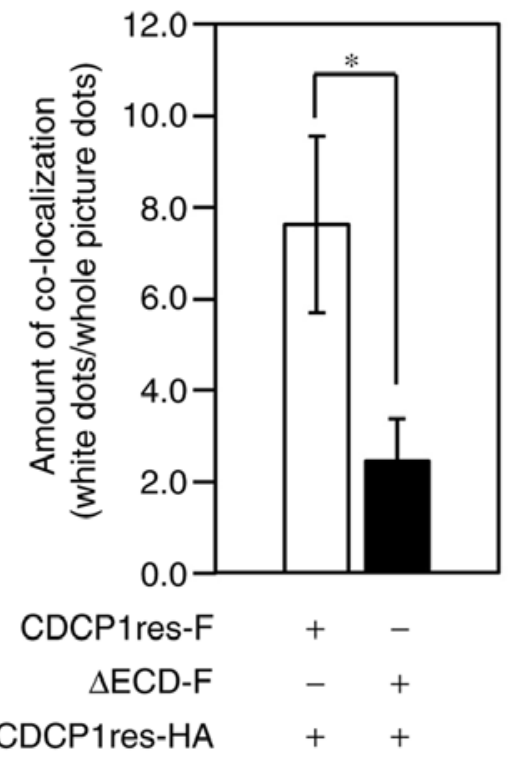

Figure 2. Localization of CDCP1 variants in A549 miCDCP1 cells. (A) CDCP1 variants were either FLAG or HA-tagged. Nuclei were stained with DAPI and the indicated antibodies: A and e, anti-FLAG (green); b and f, anti-HA (red); c and g, DAPI (blue); d and h, merge. Images were captured using a confocal microscope (magnification, $\mathrm{x} 60$ ). Scale bar $=10 \mu \mathrm{m}$. (B) Co-localization is indicated by white dots (a, b, co-localization) and the co-localization ratio was evaluated using FV10-ASW software. Scale bar $=10 \mu \mathrm{m}$. Data are presented as the mean \pm standard deviation; $\mathrm{n}=3$ for each bar; ${ }^{*} \mathrm{P}<0.05$, tukey's test. $\mathrm{CDCP} 1$, CUB domain-containing protein 1; ECD, extracellular domain; -F, FLAG-tagged; -HA, HA-tagged.

using an anti-FLAG M2 antibody. The results showed that CDCP1res-F was able to bind rMBP-CUB2; however, it was minimally bound to rMBP-CUB3 (Fig. 4B, CDCP1res-F). By contrast, $\triangle \mathrm{ECD}-\mathrm{F}$ did not bind to either rMBP fusion protein (Fig. 4B, $\triangle E C D-F$ ), and neither CDCP1 variant bound to rMBP (Fig. 4B, CDCP1res-F and $\triangle E C D-F$ ). To confirm the interaction between $\mathrm{CDCP} 1$ and purified recombinant $\mathrm{CUB}$ proteins, each CUB protein was added to the culture medium of A549 miCDCP1 cells expressing CDCP1res-HA, and the binding capacity of the CUB products was determined using immunostaining. CDCP1res-HA localization was detected at the plasma membrane and the ruffling edge, with minimal differences in CDCP1-HA expression levels between samples (Fig. 4C, inserts a, e and i). rMBP-CUB2 was co-localized with CDCP1res-HA at the plasma membrane (Fig. 4C, insert $\mathrm{g}$ ), but rMBP and rMBP-CUB3 rarely co-localized (Fig. 4C, inserts $c$ and k). The co-localization of each rMBP fusion protein with CDCP1res-HA was determined using FV10-ASW software, and is indicated by the white dotted region (Fig. 4C, inserts $\mathrm{d}, \mathrm{h}$ and $\mathrm{l}$ ). The co-localization area of rMBP-CUB2 with CDCP1res-HA $(3.32 \pm 0.46 \%)$ was greater than that of rMBP or rMBP-CUB3 with CDCP1res-HA $(0.17 \pm 0.08$ and $0.78 \pm 0.39 \%$, respectively) (Fig. 4D). These results indicated that $\mathrm{MMBP}-\mathrm{CUB} 2$ was the primary binding partner of CDCP1.

The potential rMBP-CUB2-induced inhibition of CDCP1 homophilic complex formation was also investigated. A549 miCDCP1 cells were transfected with CDCP1res-F and CDCP1res-HA expression vectors, and rMBP-CUB2 or rMBP-CUB3 proteins were added at the indicated concentrations $(0,5$ and $10 \mu \mathrm{g} / \mathrm{ml})$. No significant difference in the CDCP1 expression level was observed in these cell lines using rMBP-CUB proteins (Fig. S1A). Immunoprecipitation of A549 miCDCP1 cells with anti-FLAG and anti-HA antibodies showed the suppression of CDCP1 homophilic complex formation on addition of the CUB2 $(10 \mu \mathrm{g} / \mathrm{ml})$, but not the CUB3 domain (Fig. 4E). The two control IgG antibodies did not co-precipitate CDCP1res-FLAG or CDCP1res-HA (Fig. S1B). 
A
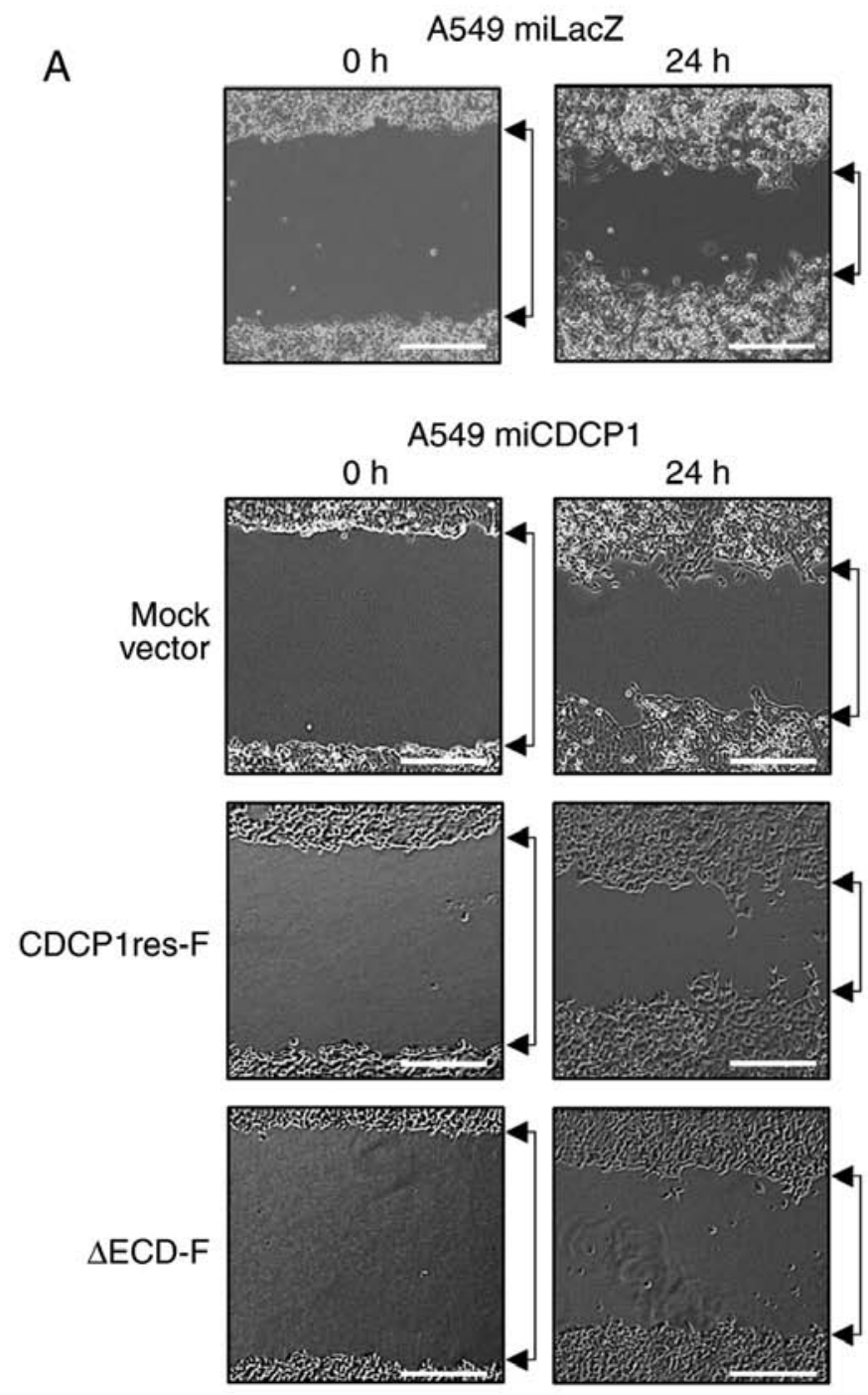

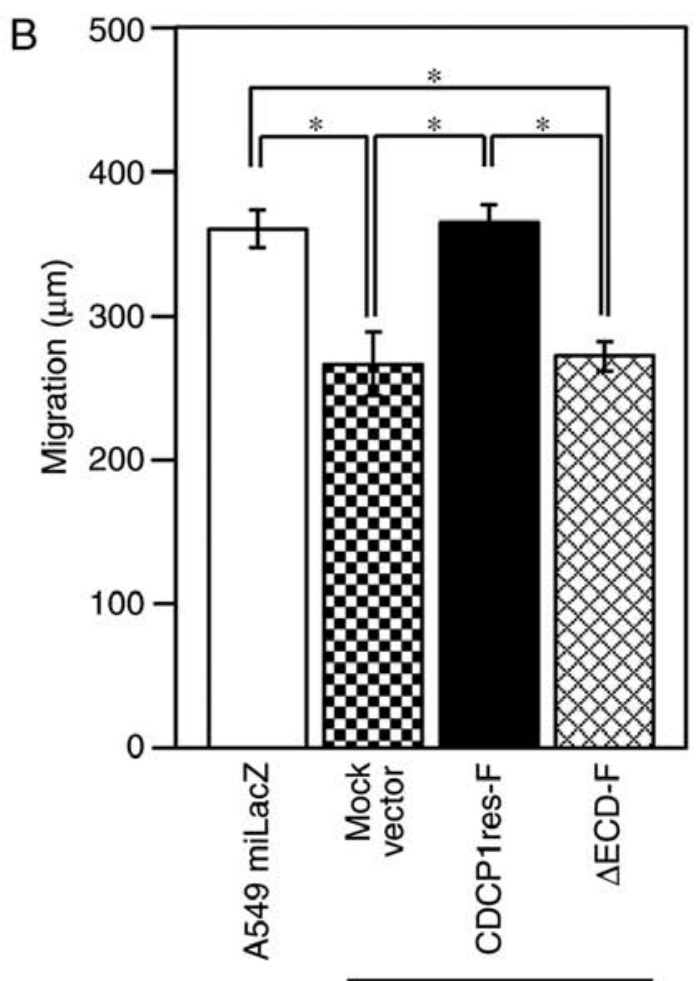

A549 miCDCP1

Figure 3. Effects of the CDCP1 ECD on cancer cell migration. (A) Migration of A549 miLacZ and A549 miCDCP1 [Mock vector (pcDNA3.1)] cells and A549 miCDCP1 CDCP1res-F and A549 miCDCP1 $\triangle$ ECD-F cells that rescued CDCP1 expression were analyzed using a scratch wound-healing assay at 0 and $24 \mathrm{~h}$. The distance between the black arrows indicates the wound width. Scale bar=500 $\mu \mathrm{m}$. Images were captured using the BZ-X710 microscope (magnification, x10), and the wound width was measured using the BZ-X Analyzer. (B) The cell migration distance was calculated by subtracting the width at $24 \mathrm{~h}$ from that at $0 \mathrm{~h}$. Mean \pm standard deviation; $\mathrm{n}=3$ for each cell; " $\mathrm{P}<0.05$, tukey's test. CDCP1, CUB domain-containing protein 1; ECD, extracellular domain; -F, FLAG-tagged.

These results suggested that the extracellular CUB2 domain of CDCP1 regulates homophilic complex formation at the plasma membrane.

The CUB2 domain of CDCP1 regulates cancer cell migration via SFK activation. Since rMBP-CUB2 effectively inhibited CDCP1 homophilic complex formation, the effect of rMBP-CUB2 on intracellular SFK-CDCP1-PKC $\delta$ signaling in parental A549 cells was investigated. Neither rMBP protein affected cell survival (Fig. S2), though SFK activation was reduced in A549 miCDCP1 cells, whose CDCP1 expression was suppressed (Fig. 5A). Notably, Tyr $^{416}$ (Y416) phosphorylation of SFK, which is an indicator of SFK activation, was suppressed following the addition of rMBP-CUB2 (Fig. 5B; SFK, lane 3), but not that of rMBP or rMBP-CUB3 (Fig. 5B, SFK, lanes 2 and 4). In addition, phosphorylation of PKC $\delta$, a downstream signal molecule of SFK, was reduced by the addition of rMBP-CUB2 (Fig. 5B PKC lane 3), but not rMBP or rMBP-CUB3 (Fig. 5B, PKCD, lanes 2 and 4). Comparable results were observed in the pancreatic cancer cell line $\mathrm{BXPC} 3$ (Fig. S3).

Finally, the effect of rMBP-CUB2 on cell migration was examined using a scratch wound-healing assay (Fig. S4). Cell migration was reduced by treatment with rMBP-CUB2 in both the A549 and BxPC3 cell lines; by contrast, rMBP and rMBP-CUB3 did not affect cell migration (Fig. 5C). These results indicated that the extracellular CUB2 domain of CDCP1 regulates cell migration by promoting SFK activation.

\section{Discussion}

In the present study, the formation of a CDCP1 homophilic complex via the CUB2 domain was demonstrated at the cell surface. This formation allows for the tighter molecular arrangement of signaling molecules at the cell surface. CDCP1 mediates the phosphorylation of PKC $\delta$ by active SFK, thus, it is 
A
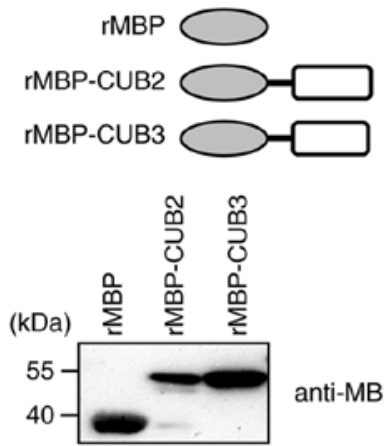

anti-MBP

B
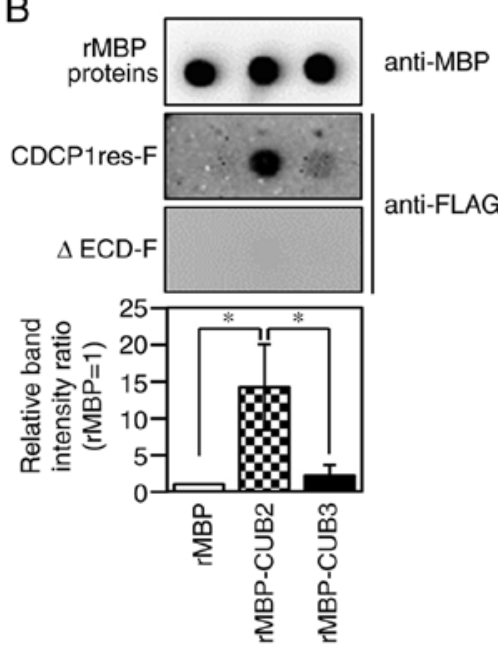

C
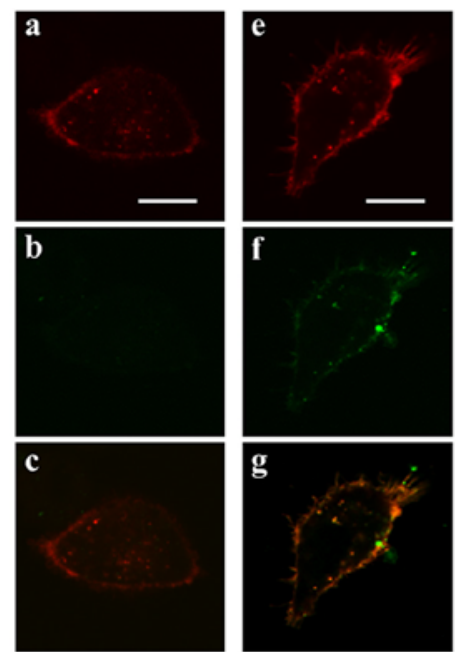

d

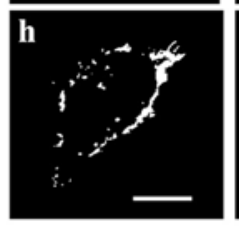

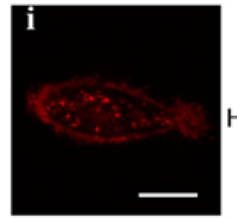

HA

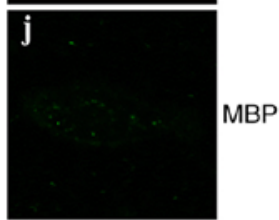

k

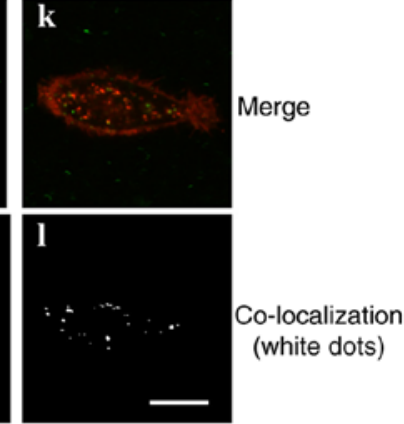

D

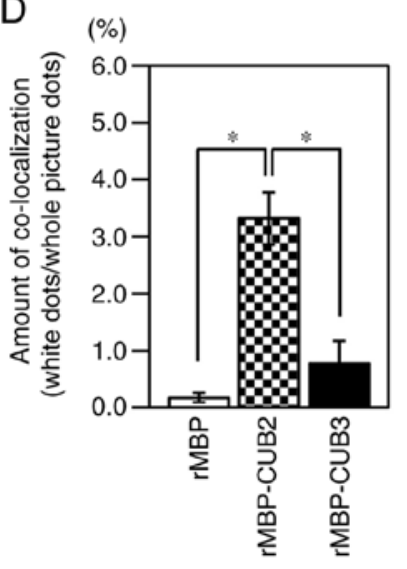

E

A549 miCDCP1 (CDCP1res-F+CDCP1res-HA)

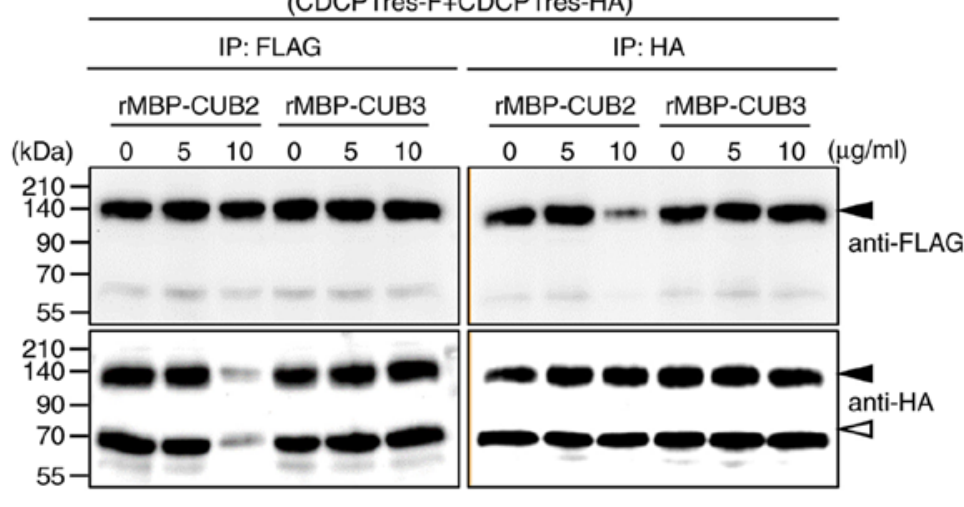

CDCP1res-HA

Figure 4. The CUB2 domain is a binding site for the CDCP1 homophilic complex. (A) Upper panel: Schematic structure of the CUB domain fused with MBP. Lower panel: Expression of each MBP construct was confirmed by western blotting using an anti-MBP antibody. (B) Far-western blotting indicating the association between MBP constructs and CDCP1 variant proteins. MBP constructs were detected using an anti-MBP antibody (rMBP proteins), and each $\mathrm{CDCP} 1$ variant was layered onto the dot-blotted rMBP proteins. The association between rMBP proteins and each CDCP1 variant (CDCP1res-F and $\triangle \mathrm{ECD}-\mathrm{F})$ was detected by western blotting with an anti-FLAG antibody, and the association ratio is shown in the bar graph. Mean \pm standard deviation; $\mathrm{n}=3$ for each bar; "P<0.05, tukey's test. (C) A549 miCDCP1 cells were transfected with CDCP1res-HA and incubated with each of the indicated rMBP proteins (rMBP, rMBP-CUB2, and rMBP-CUB3). After $24 \mathrm{~h}$, the cells were immunostained with anti-HA (a, e, and i: Red) and anti-MBP (b, f, and j: Green) antibodies. The merged image is shown in c, g, and k. Co-localization is indicated by white dots, and was determined using tFV10-ASW software (d, h, and l). Scale bar=10 $\mu \mathrm{m}$. (D) The co-localization ratio was also evaluated using FV10-ASW software; mean \pm standard deviation; $\mathrm{n}=3$ for each bar; "P<0.05, tukey's test. (E) A549 miCDCP1 cells-6 h post-transfection with CDCP1res-F and CDCP1res-HA, the medium was changed and 0,5 , or $10 \mu \mathrm{g} / \mathrm{ml} \mathrm{rMBP}-\mathrm{CUB} 2$ or rMBP-CUB3 was added; $24 \mathrm{~h}$ post-transfection, cell lysates were harvested and immunoprecipitated with anti-FLAG M2 and anti-HA antibodies. Precipitates were subjected to immunoblotting with anti-FLAG and anti-HA antibodies. The black arrowhead indicates CDCP1, and the white arrowhead indicates nonspecific bands. CDCP1, CUB domain-containing protein 1; rMBP, recombinant maltose binding protein; ECD, extracellular domain; -F, FLAG-tagged; -HA, HA-tagged.

conceivable that one of the CDCP1 molecules in the complex acts as an active SFK receptor, and that the other molecule serves as a PKC $\delta$ receptor. In the present study, co-expression of CDCP1res-F with CDCP1res-HA demonstrated that the formation of the CDCP1 complex (Fig. 1) may generate an intracellular signal (Fig. 5B, lane 1). Previous reports have 
A
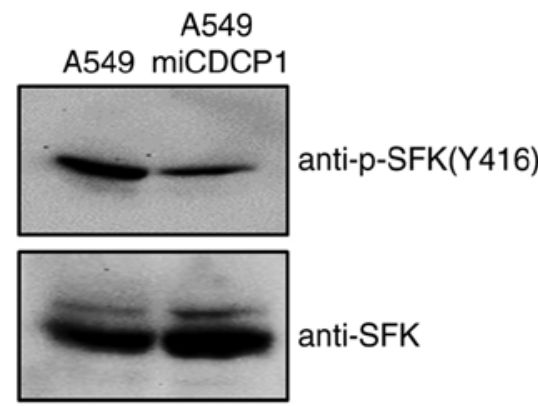

B

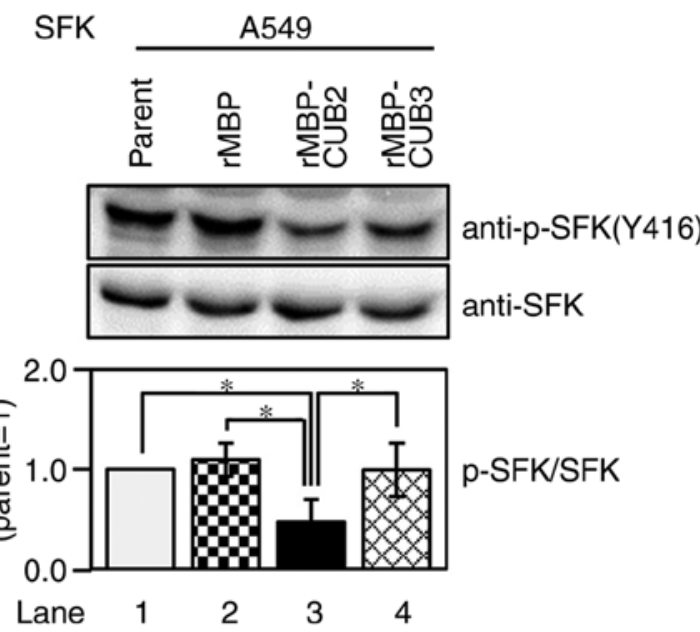

PKC $\delta$
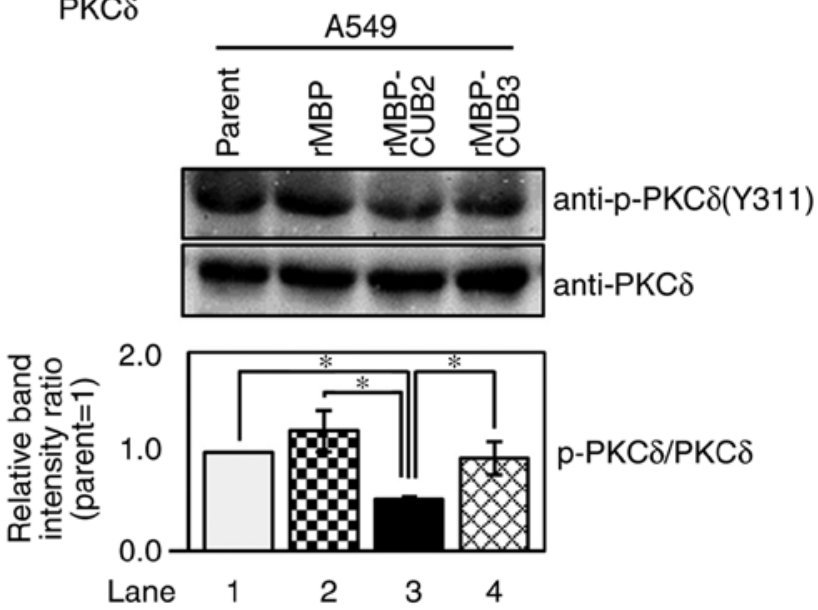
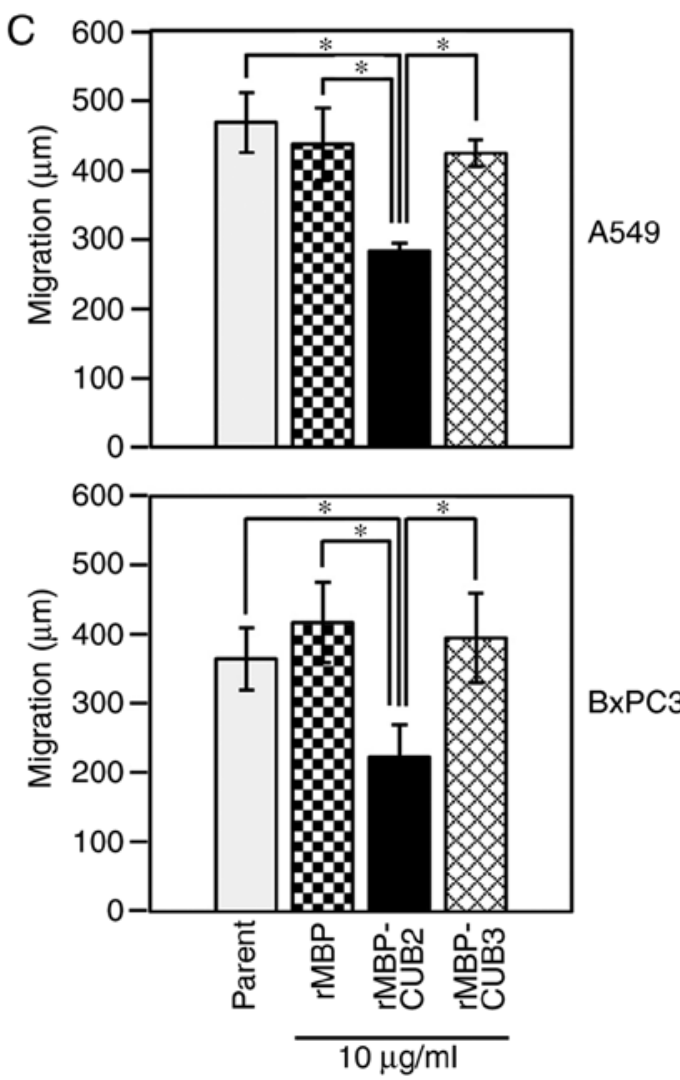

$\mathrm{BxPC3}$

Figure 5. CDCP1 CUB2 domain attenuates SFK activation and inhibits cancer cell migration. (A) Reduced CDCP1 expression attenuates SFK activation

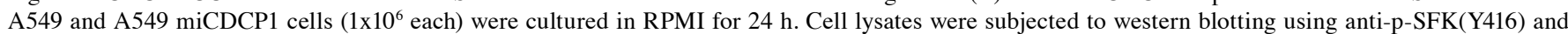
anti-SFK antibodies. (B) Effects of each rMBP fusion protein (rMBP, rMBP-CUB2 and rMBP-CUB3) on CDCP1 signaling in A549 cells. Cells were cultured with each rMBP protein for $24 \mathrm{~h}$. Lysates were subjected to immunoblotting with the indicated antibodies: Anti-p-SFK (Y416), anti-SFK, anti-p-PKC (Y311) and anti-PKCס. Adding rMBP-CUB2 alone inhibits SFK and PKC $\delta$ phosphorylation. Relative intensity ratios are shown; mean \pm standard deviation; $\mathrm{n}=3$ for each cell; " $\mathrm{P}<0.05$, tukey's test. (C) Effects of the CUB2 domain on wound-healing in A549 and BxPC3 cells. After scratching the cells and exchanging the medium, $10 \mu \mathrm{g} / \mathrm{ml}$ MBP proteins were added, and images were immediately captured $(0 \mathrm{~h})$. The scratched cells were incubated for $12(\mathrm{BxPC} 3 \mathrm{cells})$ or $24 \mathrm{~h}$ (A549 cells) in 5\% $\mathrm{CO}_{2}$, and images were captured (Fig. S4). Wound widths were measured, and migration distances were calculated by subtracting the width at 12 or $24 \mathrm{~h}$ from that at $0 \mathrm{~h}$. Mean \pm standard deviation; $\mathrm{n}=3$ for each cell line; " $\mathrm{P}<0.05$, tukey's test. CUB, complement $\mathrm{C} 1 \mathrm{r} / \mathrm{C} 1 \mathrm{~s}$, urchin embryonic growth factor, bone morphogenetic protein 1; SFK, Src family kinase; CDCP1, CUB domain-containing protein 1; p-, -phosphorylated; rMBP, recombinant maltose binding protein; $\mathrm{PKC} \delta$, protein kinase $\mathrm{C} \delta$.

indicated that cleavage between the CUB1 and CUB2 domains of the CDCP1 ECD is important for dimerization, and that the CUB2 domain may be a key region for homophilic complex formation $(18,21)$. In the present study, rMBP-CUB2 prevented CDCP1 homophilic complex formation (Fig. 4E) and decreased PKC $\delta$ phosphorylation (Fig. 5B, PKC $\delta$ lane 3); therefore, the interaction between CDCP1 molecules via the CUB2 domain is most likely a mechanism for initiating SFK-CDCP1-PKC $\delta$ signaling by clustering the receptor for active SFK and PKC $\delta$ at the cell membrane. In addition, Hooper et al (5) revealed that CDCP1 possessed 14 putative $\mathrm{N}$-glycosylation sites. The released CUB1 region of CDCP1 (30-368 residues) has a greater 
number of putative glycosylation sites than the remaining ECD (Fig. S5). Thus, it is speculated that the CUB1-containing region possessing these glycosylation sites may be a regulator for SFK-CDCP1-PKC s signaling.

SFK activation is important for SFK-CDCP1-PKCס signaling (9). CDCP1 phosphorylation is increased in metastatic cancer cells; however, the mechanism of enhanced phosphorylation is not clear. Substrates such as p130Cas and Ossa can activate SFK by associating with their phosphorylated regulatory domains $(22,23)$. CDCP1 can also activate SFK, possibly via a similar mechanism (24). In the present study, rMBP-CUB2 prevented CDCP1 homophilic complex formation (Fig. 4E) and SFK activation in cancer cells (Fig. 5B SFK lane 3). Therefore, CDCP1 accumulation at the cell membrane via the essential CUB2 domain may be required for SFK activation. The CUB2-mediated interaction of CDCP1 most likely promotes the activation of SFK by clustering inactive SFK molecules and CDCP1 within the cell membrane. It is not clear whether dimer formation is sufficient for effective signal transduction, or if oligomerization would enhance signaling activity. Furthermore, since SFK may be activated by the homophilic complex of phosphorylated CDCP1, analysis of the initial activation mechanism of SFK is required. Nonetheless, the CUB2 domain interaction between CDCP1 molecules appears to be critical for SFK activation.

In the present study, a regulatory mechanism of CDCP1 in cancer cell migration was revealed. Consistent with a previous report by Miyazawa et al (13), the CDCP1 ECD was revealed to regulate cell migration (Fig. 3). Furthermore, a positive correlation was observed between CDCP1 complex formation via the CUB2 domain and cell migration in different cancer cell lines (Figs. 5, S3 and S4). SFK-mediated CDCP1 phosphorylation is a requirement for cell migration (13). Thus, the mechanisms of CDCP1 homophilic complex formation-induced SFK activation via CUB2 may regulate cell migration mediated by intracellular SFK-CDCP1-PKCס signaling.

The extracellular CUB domain is thought to mediate interactions between various proteins (15). A number of integrins have been reported to influence CDCP1 phosphorylation (25). Moreover, a possible association between $\mathrm{N}$-cadherin (which is involved in epithelial-mesenchymal transition) and CDCP1 has also been reported (26). In addition to these previous findings, the present study delineated a role for the CUB2 domain in CDCP1 homophilic complex formation, and in the regulation of cell migration. Therefore, the CUB2 domain may be broadly involved in molecular interactions that mediate biological functions such as cell migration.

Kollmorgen et al (20) suggested that CDCP1 may form homophilic dimer complexes via its transmembrane and cytoplasmic domains. In the present study, the interaction between CDCP1res-HA and $\triangle E C D-F$ was observed (Fig. 1); however, the motility of cancer and A549 miCDCP1 cells was markedly reduced (Fig. 3). This suggests that dimer formation by transmembrane and cytoplasmic domains may regulate cancer cell functions other than migration. Therefore, further studies with CDCP1 variants are required to investigate the effects of this additional binding site on cancer cell function.

The present study suggested that the CDCP1 CUB2 domain may act as a therapeutic target. Recombinant proteins containing the CUB2 and CUB3 domains of CDCP1 are able to suppress cell migration (18), but the required domain was previously unknown. Herein, it was demonstrated that rMBP-CUB2 decreased SFK-CDCP1-PKCס signaling, and the migration of A549 and BxPC3 cell (Figs. 5, S3 and S4). Also, an interaction between rMBP-CUB3 and CDCP1res-HA was detected (Fig. 4B); however, rMBP-CUB3 did not effectively inhibit homophilic complex formation (Fig. 4E) or cancer cell migration (Fig. 5C). In addition, the comparison of amino acid sequences of each CUB domain showed low rates of homology (Fig. S6). Although, the possibility that CUB3 is critical to CDCP1 homophilic complex formation cannot be excluded, as the current data support cell migration primarily via the extracellular CUB2 domain. A previous report detailed the homophilic complex of MMP14 formed by the hemopexin-like (PEX) domain. Expression of the exogenous PEX domain also resulted in dose-dependent inhibition of cancer cell invasion via cell surface activation of proMMP-2 (27). Therefore, the present study supported that the CUB2 domain may present a novel target for the inhibition of CDCP1 homophilic complex formation and cell migration, thus contributing to the suppression of cancer cell invasion.

The present study indicated that the CUB2 domain of CDCP1 stimulates SFK activation through homophilic binding, and that it also regulates cancer cell migration. In addition, molecules that inhibit CDCP1 homologous complex formation, such as rMBP-CUB2, may be viable therapeutic candidates to combat invasive cancer.

\section{Acknowledgements}

Not applicable.

\section{Funding}

The present study was supported by a Grant-in-Aid for Scientists by the Ministry of Education, Culture, Sports, Science and Technology of Japan [JSPS KAKENHI, grant no. 15K06850 (TU)] and the National Cancer Center Research Development Fund [grant no. 23-B-24 (TU)].

\section{Availability of data and materials}

The datasets used and/or analyzed during the current study are available from the corresponding author on reasonable request.

\section{Authors' contributions}

TS, KN, TI, RS and TU made substantial contributions to conception and design or analysis and interpretation of the data. TS and TU analyzed and interpreted the data regarding CDCP1 homophilic complex formation via CUB2 domain. TS performed the examination of cell migration and SFK activation and was a primary contributor to the manuscript writing. All authors read and approved the final manuscript.

\section{Ethics approval and consent to participate}

Not applicable. 


\section{Patient consent for publication}

Not applicable.

\section{Competing interests}

The authors declare that they have no competing interests.

\section{References}

1. Hu G, Place AT and Minshall RD: Regulation of endothelial permeability by Src kinase signaling: Vascular leakage versus transcellular transport of drugs and macromolecules. Chem Biol Interact 171: 177-189, 2010.

2. Brown MT and Cooper JA: Regulation, substrates and functions of src. Biochim Biophy Acta 1287: 121-149, 1996.

3. Irby RB and Yeatman TJ: Role of Src expression and activation in human cancer. Oncogene 19: 5636-5642, 2000.

4. Summy JM and Gallick GE: Src family kinases in tumor progression and metastasis. Cancer Metastasis Rev 22: 337-358, 2003.

5. Hooper JD, Zijlstra A, Aimes RT, Liang H, Claassen GF, Tarin D, Testa JE and Quigley JP: Subtractive immunization using highly metastatic human tumor cells identifies SIMA135/CDCP1, a $135 \mathrm{kDa}$ cell surface phosphorylated glycoprotein antigen. Oncogene 22: 1783-1794, 2003.

6. Brown TA, Yang TM, Zaitsevskaia T, Xia Y, Dunn CA, Sigle RO, Knudsen B and Carter WG: Adhesion or plasmin regulates tyrosine phosphorylation of a novel membrane glycoprotein p80/gp140/CUB domain-containing protein 1 in epithelia. J Biol Chem 279: 14772-14783, 2004.

7. Bhatt AS, Erdjument-Bromage H, Tempst P, Craik CS and Moasser MM: Adhesion signaling by a novel mitotic substrate of src kinase. Oncogene 24: 5333-5343, 2005.

8. Scherl-Mostageer M, Sommergruber W, Abseher R, Hauptmann R, Ambros P and Schweifer N: Identification of a novel gene, CDCP1, overexpressed in human colorectal cancer. Oncogene 20: 4402-4408, 2001.

9. Uekita T, Jia L, Narisawa-Saito M, Yokota J, Kiyono T and Sakai R: CUB domain-containing protein 1 is a novel regulator of anoikis resistance in lung adenocarcinoma. Mol Cell Biol 27: 7649-7660, 2007.

10. Benes CH, Wu N, Elia AE, Dharia T, Cantley LC and Soltoff SP: The C2 domain of PKCdelta is a phosphotyrosine binding domain. Cell 121: 271-280, 2005.

11. Wortmann A, He Y, Christensen ME, Linn M, Lumley JW, Pollock PM, Waterhouse NJ and Hooper JD: Cellular settings mediating Src Substrates switching between focal adhesion kinase tyrosine 861 and CUB-domain-containing protein 1 (CDCP1) tyrosine 734. J Biol Chem 286: 42303-42315, 2011.

12. Dong Y, He Y, de Boer L, Stack MS, Lumley JW, Clements JA and Hooper JD: The cell surface glycoprotein CUB domain-containing protein 1 (CDCP1) contributes to epidermal growth factor receptor-mediated cell migration. J Biol Chem 287: 9792-9803, 2012.
13. Miyazawa Y, Uekita T, Hiraoka N, Fujii S, Kosuge T, Kanai Y, Nojima $Y$ and Sakai R: CUB domain-containing protein 1, a prognostic factor for human pancreatic cancers, promotes cell migration and extracellular matrix degradation. Cancer Res 70: 5136-5146, 2010.

14. Uekita T and Sakai R: Roles of CUB domain-containing protein 1 signaling in cancer invasion and metastasis. Cancer Sci 102: 1943-1948, 2011

15. Bork P and Beckmann G: The CUB domain. A widespread module in developmentally regulated proteins. J Mol Biol 231: $539-545,1993$.

16. Lee HX, Mendes FA, Plouhinec JL and De Robertis EM: Enzymatic regulation of pattern: BMP4 binds CUB domains of Tolloids and inhibits proteinase activity. Genes Dev 23: 2551-2562, 2009.

17. Romero A, Varela PF, Sanz L, Töpfer-Petersen E and Calvete JJ: Crystallization and preliminary X-ray diffraction analysis of boar seminal plasma spermadhesin PSP-I/PSP-II, a heterodimer of two CUB domains. FEBS Lett 382: 15-17, 1996.

18. Wright HJ, Arulmoli J, Motazedi M, Nelson LJ, Heinemann FS, Flanagan LA and Razorenova OV: CDCP1 cleavage is necessary for homodimerization-induced migration of triple-negative breast cancer. Oncogene 35: 4762-4772, 2016.

19. Yang L, Dutta SM, Troyer DA, Lin JB, Lance RA, Nyalwidhe JO, Drake RR and Semmes OJ: Dysregulated expression of cell surface glycoprotein CDCP1 in prostate cancer. Oncotarget 6: 43743-43758, 2015.

20. Kollmorgen G, Bossenmaier B, Niederfellner G, Häring HU and Lammers R: Structual requirements for cub domain containing protein 1 (CDCP1) and Src dependent cell transformation. PLoS One 7: e53050, 2012.

21. Casar B, He Y, Iconomou M, Hooper JD, Quigley JP and Deryugina EI: Blocking of CDCP1 cleavage in vivo prevents Akt-dependent survival and inhibits metastatic colonization through PARP1-medited apoptosis of cancer cells. Oncogene 31: 3924-3938, 2012.

22. Burnham MR, Bruce-Staskal PJ, Harte MT, Weidow CL, Ma A, Weed SA and Bouton AH: Regulation of c-SRC activity and function by the adapter protein CAS. Mol Cell Biol 20: 5865-5878, 2000

23. Tanaka M, Sasaki K, Kamata R, Hoshino Y, Yanagihara K and Sakai R: A novel RNA-binding protein, Ossa/C9orf10, regulates activity of $\mathrm{Src}$ kinase to protect cells from oxidative stress-induced apoptosis. Mol Cell Biol 22: 402-413, 2009.

24. Liu H, Ong SE, Badu-Nkansah K, Schindler J, White FM and Hynes RO: CUB-domain-containing protein 1 (CDCP1) activates Src to promotes melanoma metastasis. Proc Natl Acad Sci USA 108: 1379-1384, 2011.

25. Xia Y, Gil SG and Carter WG: Anchorage mediated by integrin alpha6beta4 to laminin 5 (epiligrin) regulates tyrosine phosphorylation of a membrane-associated $80-\mathrm{kD}$ protein. J Cell Biol 134: 724-740, 1996.

26. Miura S, Hamada S, Masamune A, Satoh K and Shimosegawa T: CUB-domain containing protein 1 represses the epithelial phenotype of pancreatic cancer cell. Exp Cell Res 321: 209-218, 2014.

27. Itoh Y, Takamura A, Ito N, Maru Y, Sato H, Suenaga N, Aoki T and Seiki M: Homophilic complex formation of MT1-MMP facilitates proMMP-2 activation on the cell surface and promotes tumor cell invasion. EMBO J 20: 4782-4793, 2001. 